\title{
On a Discrete Version of the Mohr-Mascheroni Theorem
}

\author{
Marius Munteanu, Laura Munteanu \\ Department of Mathematics, Computer Science, and Statistics, State University of New York, College at Oneonta, USA \\ Email: Marius.Munteanu@oneonta.edu, Laura.Munteanu@oneonta.edu
}

Received August 2, 2013; revised September 2, 2013; accepted September 7, 2013

Copyright (C) 2013 Marius Munteanu, Laura Munteanu. This is an open access article distributed under the Creative Commons Attribution License, which permits unrestricted use, distribution, and reproduction in any medium, provided the original work is properly cited.

\begin{abstract}
We introduce a new geometric tool called n-compass and show that the famous theorem of Mascheroni and Mohr remains valid if the traditional compass is replaced by the newly introduced tool.
\end{abstract}

Keywords: Mohr-Mascheroni Theorem; n-Compass

\section{Introduction}

The Mohr-Mascheroni theorem is one of the most interesting results concerning ruler and compass constructions (see [1-3] for a simple proof). It asserts that, as long as the objects we want to construct are points, the full power of the Euclidean tools is in fact not needed and we have the following:

Theorem 1 (Mohr-Mascheroni). Any point that can be constructed with a ruler and compass can be constructed with a compass alone.

In fact, the result has been strengthened in several ways. First, the construction can be performed in such a way that all circles have centers on an arbitrarily chosen segment (see [4,5]). In addition, as noted in [6] based on [7], the theorem can actually be generalized in a different direction by replacing the regular compass with a rusty one, i.e., a compass that can only be used to draw unit circles. It should be noted though that the authors of [7] allow for the choice of arbitrary points while performing constructions, a process is usually not allowed in pure geometric constructions (see [8] for more details). Finally, we should also mention that similar versions of the theorem have been proven in elliptic and hyperbolic geometry settings (see $[9,10]$ ).

In this note we consider the theorem above in a new context. Specifically, we introduce a new tool called $\mathrm{n}$-compass and investigate the validity of the theorem above when the classical compass is replaced by an n-compass.

\section{The n-Compass}

The main purpose for the introduction of the n-compass is to have a tool allowing for the construction of a regular n-gon. So, just like a compass can be used to draw the circle determined by two points (the center of the circle and a point on the circle), an n-compass can be used to draw the regular n-gon determined by its center and one vertex.

As a physical tool, an n-compass would consist of a central arm and $\mathrm{n}$ outside arms, all connected at a single point. The outside arms have equal lengths and make equal angles with the central one. As the angle between the central arm and one of the outside arms is modified, the other angles change by the same amount. To obtain the actual drawing of a regular n-gon, the tips of the outside arms are assumed to be connected with a flexible "chalk line". Moreover, to avoid physical constraints, the length of the central arm can be modified so as to make it possible for the construction of regular n-gons of different sizes.

By noting that an n-compass has $\mathrm{n}$ outside arms whose tips are radially $2 \pi / \mathrm{n}$ apart, it follows that a 1-compass is just a divider, while a 2-compass is a segment-doubling tool. In some sense, the classical compass can be thought of as an $\infty$-compass, although this association is rather loose. In addition, it should be noted that, unlike a compass, an n-compass cannot be swung; a regular n-gon is drawn by simply placing the tool on paper and considering the trace of the "chalk line".

\section{The Main Result}

In defining what it means for a point to be constructible (with any tools), one usually thinks of a process consist- 
ing of several steps at the end of which the point in question is obtained as an intersection of objects constructed in one of the previous steps. To avoid any ambiguities in the definition, one must impose that no arbitrary points be used at any step. Hence, we must have a starter set in order to be able to get off the ground and this set should consist of at least two points. This is actually the starter set one has in mind in the statement of the MohrMascheroni theorem. However, it should be noted that the aforementioned theorem remains valid for any starter set consisting of at least two points.

We claim that the following version of the MohrMascheroni theorem, stated in terms of starter sets, holds in the context of n-compass constructions.

Theorem 2. Given a set $S$ consisting of at least two points, any point that can be constructed with ruler and $n$-compass $(n>2)$ and starter set $S$ can also be constructed with just an n-compass and the same starter set.

Proof: Clearly, the theorem follows as long as we could find a way to replicate a ruler. Since the sides of an $\mathrm{n}$-gon consist of straight line segments, we will use these segments to create our ad-hoc ruler. In particular, given any two points $\mathrm{X}$ and $\mathrm{Y}$, as a first step, we need to be able to construct a regular n-gon such that the line is determined by two of its vertices contains $\mathrm{X}$ and $\mathrm{Y}$. However, this is not enough since one actually has to be able to extend the segment $\mathrm{XY}$ in both directions, as needed. So our approach consists of two steps represented by the following constructions:

Construction 1. Given two points X, Y, by using only an n-compass, it is possible to construct a regular n-gon such that the extension of one of the sides of this polygon contains both $\mathrm{X}$ and $\mathrm{Y}$.

Construction 2. Given two consecutive vertices $P_{1}$ and $\mathrm{P}_{2}$ of a regular n-gon (given with together with its center), it is possible to extend segment $\mathrm{P}_{1} \mathrm{P}_{2}$ indefinitely in both directions by using only an n-compass.

For Construction 1, let us first construct the regular n-gon centered at $\mathrm{Y}$ with "radius" $\mathrm{YX}$ and denote consecutive vertices of this n-gon (in clockwise orientation) by $X=X_{1}, X_{2}, \ldots, X_{n}$. Similarly, the vertices of the regular n-gon centered at $X$ with "radius" $X Y$ will be denoted by $\mathrm{Y}=\mathrm{Y}_{1}, \mathrm{Y}_{2}, \ldots, \mathrm{Y}_{\mathrm{n}}$, but this time the notation is in counterclockwise orientation (see Figure 1). With center at $X_{2}$, we now draw the regular n-gon with "radius" $X_{2} X$ and label its vertices in a counterclockwise manner by $X$ $=Z_{1}, Z_{2}, \ldots, Z_{n}$. We claim that $Z_{2}$ is on line $X Y$ and hence $X$ and $Y$ are on line $Z_{1} Z_{2}$. Indeed, if $n$ is equal to 6 , then $X_{2}$ coincides with $Y_{2}, Z_{2}$ coincides with $Y$, and we are done. If $\mathrm{n}$ is different from 6 , then $X_{2}$ and $Y_{2}$ are different and we can consider the parallel to line $Y_{1} Y_{2}$ through $X_{2}$ and denote its intersection with $X_{1} Y_{1}$ by W. Note that we are only referring to this line in order to prove that Construction 1 works, we do not need to con-

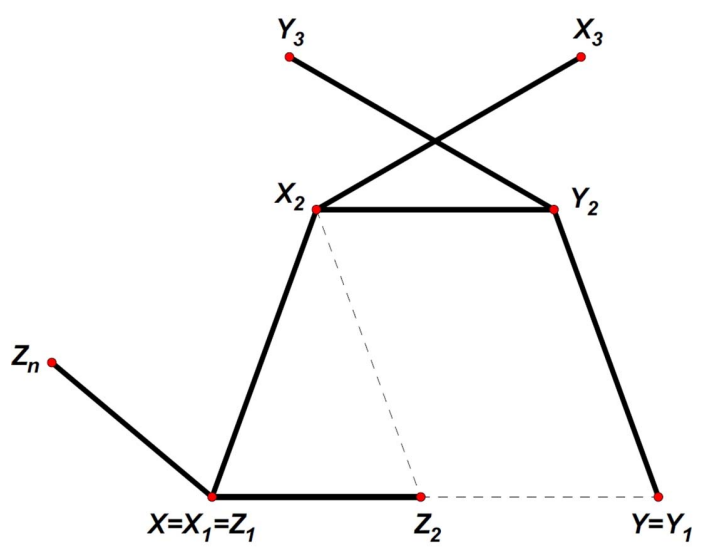

Figure 1. "Connecting” $\mathrm{X}$ and $\mathrm{Y}$.

struct it. In fact, we will show that $\mathrm{W}$ coincides with $\mathrm{Z}_{2}$, which will finish the proof. To this end, note that lines $X_{2} Y_{2}$ and $X_{1} Y_{1}$ are parallel due to symmetry. Hence, the quadrilateral $\mathrm{Y}_{1} \mathrm{Y}_{2} \mathrm{X}_{2} \mathrm{~W}$ is in fact a parallelogram, implying that the segments $\mathrm{Y}_{1} \mathrm{Y}_{2}$ and $\mathrm{X}_{2} \mathrm{~W}$ are congruent. But $X_{1} X_{2}$ and $Y_{1} Y_{2}$ are also congruent as equal sides of regular n-gons with the same "radius". So we have that the segments $\mathrm{X}_{2} \mathrm{X}_{1}, \mathrm{X}_{2} \mathrm{Z}_{1}$, and $\mathrm{X}_{2} \mathrm{~W}$ are congruent and

$$
m\left(\measuredangle Z_{1} X_{2} W\right)=\pi-2 \frac{(n-2) \pi}{2 n}=\frac{2 \pi}{n} .
$$

Now clearly $\mathrm{W}$ and $\mathrm{Z}_{2}$ must coincide since the relation above shows that $\mathrm{W}$ is the rotation of $Z_{1}$ in $X_{2}$ through $2 \pi / n$. It should be noted that the regular $n$-gon centered at $\mathrm{X}$ is not needed in the actual construction of $\mathrm{Z}_{2}$. We only used side $Y_{1} Y_{2}$ of this $n$-gon to prove that $X, Y$, and $Z_{2}$ are collinear.

For Construction 2, let $\mathrm{Q}_{1}$ be the center of the regular n-gon having $\mathrm{P}_{1} \mathrm{P}_{2}$ as one of its sides. Throughout this proof we will assume that the indexing order among the vertices of any regular n-gon corresponds to the counterclockwise orientation, considering now the regular n-gon centered at $\mathrm{P}_{1}$ with "radius" $\mathrm{P}_{1} \mathrm{Q}_{1}$. Finally, let us also construct the regular n-gon centered at $\mathrm{Q}_{2}$ with "radius" $\mathrm{Q}_{2} \mathrm{P}_{1}$. If we denote the vertices of this $\mathrm{n}$-gon by $\mathrm{R}_{1}$, $R_{2}, \ldots, R_{n}$, by relabeling $P_{1}$ as $R_{2}$, we claim that $R_{1}$ is on line $P_{1} P_{2}$ (see Figure 2). Thus, the segment $P_{1} R_{1}$ extends the segment $\mathrm{P}_{1} \mathrm{P}_{2}$ on the $\mathrm{P}_{1}$-side of $\mathrm{P}_{1} \mathrm{P}_{2}$. Clearly, this construction can be continued (on both sides of $\mathrm{P}_{1} \mathrm{P}_{2}$ ) to obtain an arbitrarily long extension of $\mathrm{P}_{1} \mathrm{P}_{2}$.

To prove the claim, notice that

$$
\mathrm{m}\left(\measuredangle \mathrm{Q}_{1} \mathrm{P}_{1} \mathrm{P}_{2}\right)=\mathrm{m}\left(\measuredangle \mathrm{Q}_{2} \mathrm{P}_{1} \mathrm{R}_{1}\right)=\frac{(\mathrm{n}-2) \pi}{2 \mathrm{n}}
$$

and that

$$
\mathrm{m}\left(\measuredangle \mathrm{Q}_{2} \mathrm{P}_{1} \mathrm{Q}_{1}\right)=\frac{2 \pi}{\mathrm{n}} .
$$




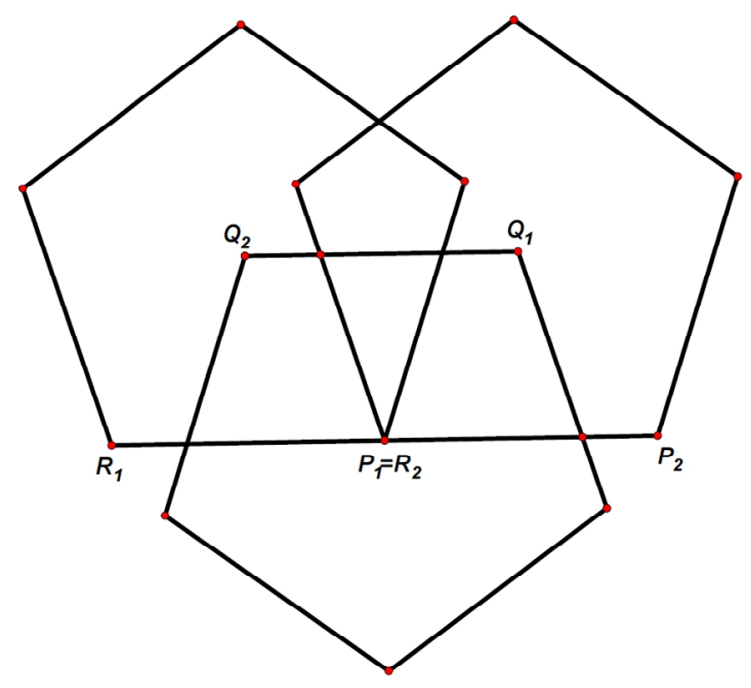

Figure 2. Extending a side.

Since the sum of the measures of the three angles above is $\pi$, it follows that $\mathrm{R}_{1}, \mathrm{P}_{1}$, and $\mathrm{P}_{2}$ are collinear points, as claimed.

\section{REFERENCES}

[1] L. Mascheroni, "Geometrie du Compas," Monom, 1980.

[2] G. Mohr, "Euclides Danicus," Copenhagen, 1928.
[3] N. Hungerbuhler, "A Short Elementary Proof of the Mohr-Mascheroni Theorem," American Mathematical Monthly, Vol. 101, No. 8, 1994, pp. 784-797. http://dx.doi.org/10.2307/2974536

[4] A. Avron, "Theorems on Strong Constructibility with a Compass Alone," Journal of Geometry, Vol. 30, No. 1, 1987, pp. 28-35. http://dx.doi.org/10.1007/BF01223260

[5] A. Avron, "On Strict Strong Constructibility with a Compass Alone," Journal of Geometry, Vol. 38, No. 1-2, 1990, pp. 12-15. http://dx.doi.org/10.1007/BF01222890

[6] V. Pambuccian, "Axiomatizing Geometric Constructions," Journal of Applied Logic, Vol. 6, No. 1, 2008, pp. 24-46. http://dx.doi.org/10.1016/j.jal.2007.02.001

[7] Z. Zhang, L. Yang and X. R. Hou, "What Can We Do with Only a Pair of Rusty Compasses?" Geometriae Dedicata, Vol. 38, 1991, pp. 137-150. http://dx.doi.org/10.1007/BF00181213

[8] V. J. Baston and F. A. Bostok, "On the Impossibility of Ruler-Only Constructions," Proceedings of the American Mathematical Society, Vol. 110, No. 4, 1990, pp. 10171025.

http://dx.doi.org/10.1090/S0002-9939-1990-1034882-3

[9] D. Fog, "Om Konstruktion Med Passeren Alene," Mathematisk Tidsskrift A, 1935, pp. 16-24.

[10] V. S. Martynenko, "Mascheroni's Theorem in Lobachevski Geometry," Doklady Akademii Nauk Ukrainskoj SSR, Ser A, Vol. 1, 1982, pp. 22-27. 